\title{
Optimization of the ultra-flattened normal dispersion in photonic crystal fibers infiltrated with olive oil for supercontinuum generation
}

\author{
Hieu Le Van, ${ }^{* 1}$ Bien Chu Van, ${ }^{1}$ Dinh Thuan Bui, ${ }^{2}$ Trung Le Canh, ${ }^{2}$ Quang Ho Dinh, ${ }^{3}$ Dinh Nguyen $\operatorname{Van}{ }^{4}$ \\ ${ }^{1}$ Faculty of Natural Sciences, Hong Duc University, 565 Quang Trung Street, Thanh Hoa City, Vietnam, \\ ${ }^{2}$ School of natural sciences education, Vinh University, 182 Le Duan Street, Vinh city, Vietnam \\ ${ }^{3}$ School of Chemistry, Biology and Environment, Vinh University, 182 Le Duan Street, Vinh City, Vietnam, \\ ${ }^{4}$ Vietnam Naval Academy, 30 Tran Phu Street, Nha Trang City, Vietnam
}

Received October 25, 2020; accepted January 11, 2021; published March 14, 2021

\begin{abstract}
This paper proposes a pure silica photonic crystal fiber (PCF), having its core infiltrated with olive oil, which allows achieving an ultra-flattened normal dispersion regime. As a result, the optimization processes allows us to achieve an ultra-flat normal dispersion in the range of over $682 \mathrm{~nm}$ within the wavelength range from 1446 to 2128 $\mathrm{nm}$. Besides, the nonlinear coefficient of the selected PCF structure is extremely high $\left(9.54 \times 10^{9} \mathrm{~W}^{-1} \mathrm{~km}^{-1}\right.$ at $\left.1550 \mathrm{~nm}\right)$. The proposed PCF structure could be very helpful in investigating the supercontinuum generation which has many potential applications in various promising areas such as spectroscopy, medical diagnostics, etc.
\end{abstract}

Supercontinuum (SC) generation in photonic crystal fiber (PCF) has been the topic of extensive studies over the past decade due to its unique novel properties and its important applications in numerous promising areas, for instance, optical communications, frequency metrology and optical coherence tomography $[1 \div 4]$. SC generation is typically achieved by launching ultrashort laser pulses near the zero dispersion point into a highly nonlinear fiber. Thus, large effective nonlinearity is essential for the broadband of a flat SC spectrum. The only method to increase the value of a nonlinear coefficient of the PCF with a silica core is reducing the effective core area. However, this causes a problem that a small effective core area will lead to coupling difficulty. Besides that, silica glass is not transparent in the mid-infrared (IR) range and the spectral bandwidth is also limited in the visible to near-IR wavelength range. Therefore, the improvement of SC generation sources of PCFs from a non-silica, highly nonlinear material becomes significant.

As a typical solution, SC sources have been developed

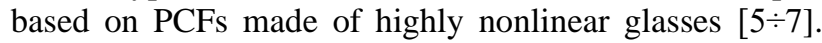
These materials revolve further broadening of spectral coverage including mid-IR wavelengths. Because of the highly nonlinear refractive indices of these materials, the $\mathrm{SC}$ spectrum is expected to generate significantly shorter propagation scales. Also, broader and smoother SC will be generated with the same laser parameters. However, a highly nonlinear material will cause high costs and also lead to a complex fabrication process.
Another method to achieve higher values of nonlinear material is using hollow-core PCFs filled with liquids [8][11]. Owing to the higher nonlinear refractive index of liquids in comparison to solids $[12 \div 13]$, it is possible to observe interesting nonlinear phenomena, such as SC generation with a lower peak power than in classical solid fiber [9]. Furthermore, the optical absorption of a liquid core in the region of visible and near-IR wavelength is relatively low [14]. Thus, it is also expected that liquids core PCFs, whose lengths are about a few centimeters, would be employed for generating SC $[9 \div 10]$. However, high nonlinearity liquids are usually highly toxic, which severely limits their potential application and also leads issues related to drawing fibers based on exotic, toxic, carcinogenic, explosive, and expensive soft glasses, as well as thermal matching between core and cladding.

In this letter, we propose and analyze for the first time a fused-silica PCF having its core infiltrated with olive oil, for an ultra-flat normal dispersion region. The guiding properties of fundamental mode including dispersion, mode area, and nonlinear coefficients are considered numerically. At first, we carry out optimizations of dispersion properties via modifying photonic parameters, i.e., the lattice constant and size of an air hole to obtain ultra-flattened dispersion inside the normal region. And then, the optical properties of such an optimal PCF structure are analyzed in detail.

We have chosen olive oil because its nonlinear refractive index is extremely high rather than those for other liquids and solids. Indeed, the olive oil has a nonlinear refractive index value of $2.28 \times 10^{-11} \mathrm{~m}^{2} / \mathrm{W}$ at $632.8 \mathrm{~nm}$ [15], while it equals $310 \times 10^{-20} \mathrm{~m}^{2} / \mathrm{W}$ for carbon disulfide or equals $2.6 \times 10^{-20} \mathrm{~m}^{2} / \mathrm{W}$ for fused silica at 530 $\mathrm{nm}$ [12]. This is promising for broadband SC all-fiber sources with only few millimeters. One special thing when we compare it to high nonlinear liquids is that olive oil is non-toxic. This is very important in reality because it will overcome the disadvantages that high nonlinear liquids bring.

*E-mail: levanhieu@hdu.edu.vn 
The cross-section of the geometrical structure schematic of the modeled PCF is presented in Fig. 1. Here, we model the PCF with a fused-silica cladding which consists of seven air-hole rings arranged in a regular hexagonal lattice shape. This cross-sectional structure is defined by the lattice pitch $\Lambda$ and air holes diameter in the cladding $d$. The linear filling factor of the cladding is defined as $f=d / \Lambda$ and it used a constant filling factor for all rings to simplify future fiber development. The central air hole is much bigger and fulfils the relation: $D_{c}=2 \Lambda-1.4 d$, to ensure the technological feasibility of the considered structures. We also assume that the central hole is filled with olive oil.

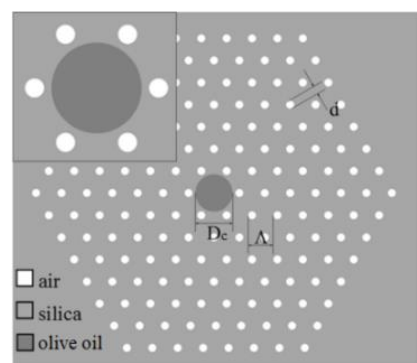

Fig. 1. Schematic of the modeled PCF structure, where Dc is the diameter of the liquid-filled core.

The refractive index characteristics for modeling obey the Sellmeier equation as the below formula:

$$
n(\lambda)=\sqrt{A_{1}+\frac{B_{1} \lambda^{2}}{\lambda^{2}-C_{1}}+\frac{B_{2} \lambda^{2}}{\lambda^{2}-C_{2}}+\frac{B_{3} \lambda^{2}}{\lambda^{2}-C_{3}}}
$$

where $B_{i}$ and $C_{i}$ are the Sellmeier coefficients and $\lambda$ is the operating wavelength in $\mu \mathrm{m}$. The Sellmeier's coefficients for fused-silica and olive oil are introduced in Table 1.

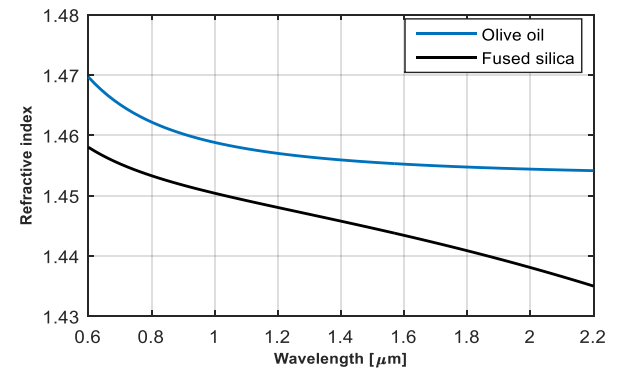

Fig. 2. Real part of the refractive index of olive oil and fused silica.

Table 1. Sellmeier's coefficients for the material used.

\begin{tabular}{|c|c|c|}
\hline Coefficient & Fused silica [16] & Olive oil [17] \\
\hline $\mathrm{A}_{1}$ & 1 & 1 \\
\hline $\mathrm{B}_{1}$ & 0.6694226 & 1.111 \\
\hline $\mathrm{B}_{2}$ & 0.4345839 & 0 \\
\hline $\mathrm{B}_{3}$ & 0.8716947 & 0 \\
\hline $\mathrm{C}_{1}\left[\mu \mathrm{m}^{2}\right]$ & 0.0044801 & 0.012321 \\
\hline $\mathrm{C}_{2}\left[\mu \mathrm{m}^{2}\right]$ & 0.013285 & 0 \\
\hline $\mathrm{C}_{3}\left[\mu \mathrm{m}^{2}\right]$ & 95.341482 & 0 \\
\hline
\end{tabular}

It can be seen that the refractive index of olive oil is higher than that of fused-silica glass as shown in Fig. 2. Therefore, the guiding mechanism is derived by total internal reflection along the PCF.

We have divided linear simulations into two parts:

1. Analyze the function of the lattice pitch and filling factor of the PCF structure;

2. Detailed analysis of the structures with optimized dispersion.

Looking for the optimal structure of PCF we consider the structures having the lattice pitch $\Lambda$ changing from 1.5 to 2.5 with a step of 0.5 and the filling factor changing from 0.3 to 0.6 with a step of 0.025 . As a result, 39 simulation runs were conducted at this stage. The optimization criteria aimed at SC generation at selected wavelengths of $1.55 \mu \mathrm{m}$ and followed the flatness of dispersion (the difference between maximum and minimum dispersion values), and spectral bandwidth within the appropriate dispersion variability range.
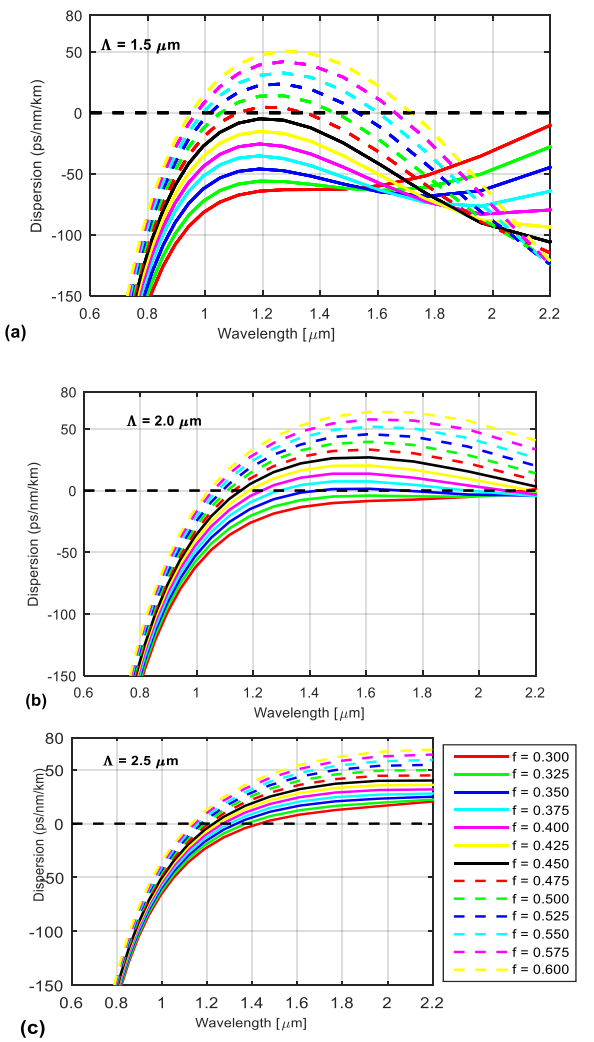

Fig. 3. Characteristics of the fundamental mode dispersion for different lattice pitch $\Lambda$.

Figure 3 shows the characteristics of dispersion for the fundamental mode in the $0.5 \div 2.2 \mu \mathrm{m}$ wavelength range. For a certain $\Lambda$ value, increasing the filling factor leads not only to an increased difference between maximum and minimum dispersion values but also to the dispersion bandwidth. Meanwhile, for a certain $f$, the characteristics are shifted toward longer waves and flattened with increasing $\Lambda$. The same applies to ZDWs. In the case of the PCF structure with a lattice pitch equal to $2.5 \mu \mathrm{m}$, the 
maximum dispersion is greater than zero, which means there is both normal and anomalous dispersion. On the contrary, dispersion exists only in the normal dispersion range or a part that exist in both ranges.

Finally, from the preliminary calculations that were conducted to achieve ultra-flattened dispersions and their wide bandwidth, we selected the PCF with a lattice pitch equal to $2.0 \mu \mathrm{m}$ and a filling factor of 0.325 . This fiber has the optimum dispersion characteristics, because of the achieved flatness and largest bandwidth in the normal dispersion region. The result illustrates that the proposed PCF allows obtaining ultra-flat normal dispersion over the $682 \mathrm{~nm}$ wavelength range from 1446 to $2128 \mathrm{~nm}$, where the dispersion changed from -6.078 to $-4.773 \mathrm{ps} / \mathrm{nm} / \mathrm{km}$.

Figure 4 (a) shows the comparison of dispersion characteristics of the fundamental mode as a wavelength function of the designed PCF and the PCF of a solid core with fused silica glass. The fiber of optimal size, i.e. for fused-silica core has existing two ZDWs; the dispersion at $1.55 \mu \mathrm{m}$ wavelength equals $-12.123773 \mathrm{ps} / \mathrm{nm} / \mathrm{km}$, while the proposed fiber exists in all normal dispersion in this range, and the dispersion at $1.55 \mu \mathrm{m}$ wavelength equals $4.773 \mathrm{ps} / \mathrm{nm} / \mathrm{km}$.
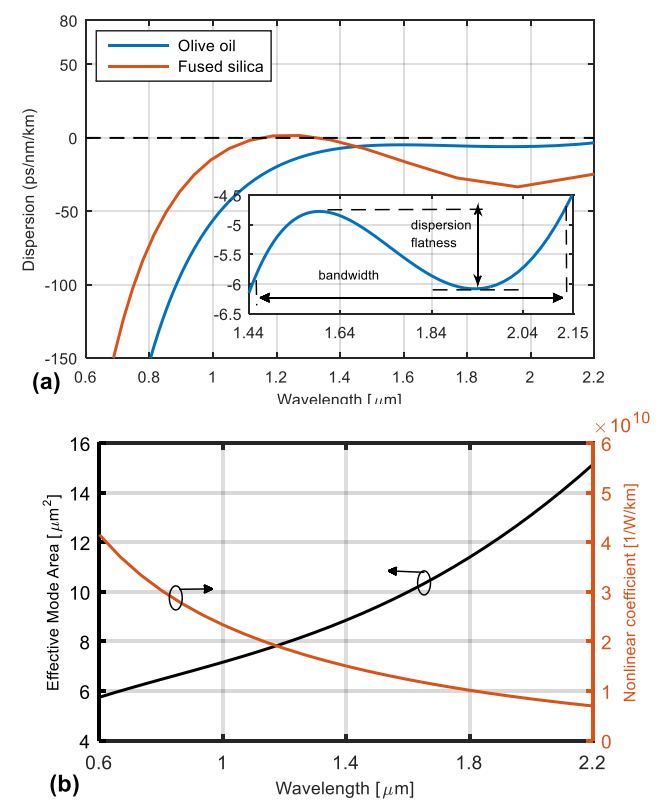

Fig. 4. (a) Dispersion characteristics of the PCF with a core filled with air and with olive oil; (b) calculated effective mode area, nonlinear refractive index of the designed PCF.

The effective mode area and nonlinear coefficient of this structure are depicted in Fig. 4 (b). The modal area of the fundamental mode increases almost linearly with the wavelength. For a wavelength at $0.5 \mu \mathrm{m}$, the modal area equals $5.33659 \mu \mathrm{m}^{2}$, while at a $1.55 \mu \mathrm{m}$ wavelength, the modal area equals $9.68821 \mu \mathrm{m}^{2}$.

Meanwhile, the nonlinear coefficient $\gamma$ of the proposed $\mathrm{PCF}$ is calculated by the following equation:

$$
\gamma=\frac{2 \pi}{\lambda} \frac{n_{2}}{A_{e f f}}
$$

where $n_{2}$ is the nonlinear refractive index of olive oil $\left(n_{2}=\right.$ $2.28 \times 10^{-11} \mathrm{~m}^{2} / \mathrm{W}$ at $\left.632.8 \mathrm{~nm}[15]\right)$ and $A_{\text {eff }}$ denotes the effective mode area. The designed fiber structure has an extremely high nonlinear coefficient whose values can be higher than $4.15 \times 10^{10} \mathrm{~W}^{-1} \mathrm{~km}^{-1}$. At a $1.55 \mu \mathrm{m}$ wavelength, the nonlinear coefficient reaches $9.54 \times 10^{9} \quad \mathrm{~W}^{-1} \cdot \mathrm{km}^{-1}$. Thus, with an extremely highly nonlinear one, it is also expected that olive oil core PCFs, whose lengths are about a few millimeters, could be used for generating SC. The designed PCF would be very useful for studying SC generation which has many potential applications in different fields such as spectroscopy, frequency metrology, optical coherence tomography, medical diagnostics, chemical detection, etc.

This work was supported by the project 796/2019/ HĐKHCN-ĐTKHCN.

\section{References}

[1] J.M. Dudley, G. Genty, S. Coen, Rev. Mod. Phys. 78, 1135 (2006).

[2] T. Udem, R. Holzwarth, T.W. Hänsch, Nature 416, 233 (2002).

[3] S. Moon, D.Y. Kim, Opt. Expr. 14, 11575 (2006).

[4] G.P. Agrawal, Nonlinear Fiber Optics (Oxford: Academic Press 2013).

[5] V.R.K. Kumar, A.K. George, J.C. Knight, P.S.J. Russell, Opt Expr. 11, 2641 (2003).

[6] R. Buczynski, H.T. Bookey, D. Pysz, R. Stepien, I. Kujawa, J.E. McCarthy, A.J. Waddie, A.K. Kar, M.R. Taghizadeh, Laser Phys. Lett. 7, 666 (2010).

[7] F.G. Omenetto, N.A. Wolchover, M.R. Wehner, M. Ross, A. Efimov, A.J. Taylor, V.V.R.K. Kumar, A.K. George, J.C. Knight, N.Y. Joly, P.St.J. Russell, Opt. Expr. 14, 4928 (2010).

[8] H. L.Van, V.C. Long, H.T. Nguyen, A.M. Nguyen, R. Buczyński, R. Kasztelanic, Laser Physics 28, 115106 (2018).

[9] J. Pniewski, T. Stefaniuk, H.L. Van, V.C. Long, L.C. Van, R. Kasztelanic, G. Stępniewski, A. Ramaniuk, M. Trippenbach, R. Buczynski, Appl. Opt. 55, 5033 (2016).

[10] H.D. Quang et al., Appl. Opt. 57(15), 1559 (2018).

[11] M. Chemnitz, M. Gebhardt, C. Gaida, F. Stutzki, J. Kobelke, J. Limpert, A. Tünnermann, M.A. Schmidt, Nat. Commun. 842 (2017).

[12] S. Kedenburg, A. Steinmann, R. Hegenbarth, T. Steinle, H. Giessen, Appl. Phys. B 117803 (2014).

[13] E. Sani, A.Dell'Oro, Opt. Mater. 60, 137 (2016).

[14] S.T. Wu, J. Appl. Phys. 84, 4462 (1998).

[15] Z. Mousavi, B. Ghafary, M.H. Majles, J. Molec. Liquids 285, 444 (2019).

[16] Refractive Index Info: https://refractiveindex.info.

[17] I. Bodurov, I. Vlaeva, M. Marudova, T. Yovcheva, K. Nikolova, T. Eftimov, V. Plachkova, Bulgarian Chem. Comm. 45(Issue B), 81 (2013) 